\title{
Impact of antibiotic prophylaxis for intrathecal baclofen pump surgery in pediatric patients
}

\author{
I-Wen Pan, PhD,, ${ }^{1,2}$ Grace M. Kuo, PharmD, PhD, ${ }^{3}$ Thomas G. Luerssen, MD,, ${ }^{1,2}$ and \\ Sandi K. Lam, MD, MBA ${ }^{1,2}$
}

'Department of Neurosurgery, Baylor College of Medicine, and 'Division of Pediatric Neurosurgery, Texas Children's Hospital, Houston, Texas; and ${ }^{3}$ Skaggs School of Pharmacy and Pharmaceutical Sciences, University of California, San Diego, La Jolla, California

OBJECT This study explored antibiotic prophylaxis (AP) in pediatric patients undergoing intrathecal baclofen pump (ITBP) surgery and factors associated with perioperative AP compliance with clinical guidelines.

METHODS Data were obtained from the Pediatric Health Information System. The study cohort comprised patients who underwent ITBP surgery within 3 days of admission, between July 1, 2004, and March 31, 2014, with a minimum prior screening period and follow-up of 180 days. Exclusion criteria were prior infection, antibiotic use within 30 days of admission, and/or missing financial data. Chi-square tests and multivariate logistic regressions were used to determine factors associated with compliance with AP guidelines in ITBP surgeries.

RESULTS A total of 1,534 patients met the inclusion criteria; $91.5 \%$ received AP and $37.6 \%$ received dual coverage or more. Overall bundled compliance comprised 2 components: 1) perioperative antibiotic administration and 2) <24-hour postoperative antibiotic course. The most frequently used antibiotics in surgery were cefazolin $(n=873,62.2 \%)$ and vancomycin $(n=351,25 \%$ ). Documented bundled AP compliance rates were $70.2 \%, 62.0 \%, 66.0 \%$, and $55.2 \%$ in West, South, Midwest, and Northeast regions of the US, respectively. Compared with surgeries in the Northeast, procedures carried out in the West (OR 2.0, 95\% Cl 1.4-2.9, p < 0.001), Midwest (OR 1.6, 95\% Cl 1.1-2.3, p = 0.007), and South (OR 1.5, 95\% Cl 1.1-2.0, $p=0.021)$ were more likely to have documented AP compliance. Black $(\mathrm{OR} 0.74,95 \% \mathrm{Cl} 0.55-$ $1.00, p=0.05$ ) and Hispanic (OR 0.63, 95\% Cl 0.47-0.86, $p=0.004$ ) patients were less likely to have documented AP compliance in ITBP surgeries than white patients. There were no significant differences in compliance rate by age, sex, type of insurance, and diagnosis. AP process measures were associated with shorter length of stay, lower hospitalization costs, and lower 6 -month rates of surgical infection/complication. One of the 2 noncompliance subgroups, missed preoperative antibiotic administration, was correlated with a significantly higher 6-month surgical complication/infection rate $(27.03 \%)$ compared with bundled compliance $(20.00 \%, p=0.021)$. For the other subgroup, prolonged antibiotic use $>24$ hours postoperatively, the rate was insignificantly higher $(22.00 \%, p=0.368)$. Thus, of direct relevance to practicing clinicians, missed preoperative antibiotics was associated with $48 \%$ higher risk of adverse complication/infection outcome in a 6-month time frame. Adjusted hospitalization costs associated with baclofen pump surgery differed significantly ( $p$ $<0.001$ ) with respect to perioperative antibiotic practices: 22.83, 29.10, 37.66 (× 1000 USD) for bundled compliance, missed preoperative antibiotics, and prolonged antibiotic administration, respectively.

CONCLUSIONS Significant variation in ITBP antibiotic prophylaxis was found. Documented AP compliance was associated with higher value of care, showing favorable clinical and financial outcomes. Of most impact to clinical outcome, missed preoperative antibiotics was significantly associated with higher risk of 6-month surgical complication/infection. Prolonged antibiotic use was associated with significantly higher hospital costs compared with those with overall bundled antibiotic compliance. Future research is warranted to examine factors associated with practice variation and how AP compliance is associated with outcomes and quality, aiming for improving delivery of care to pediatric patients undergoing ITBP procedures.

http://thejns.org/doi/abs/10.3171/2015.9.FOCUS15385

KEY WORDS intrathecal baclofen pump; antibiotics; antibiotic prophylaxis; compliance; pediatric

ABBREVIATIONS AP = antibiotic prophylaxis; ASHP = American Society of Health-System Pharmacists; ICD-9-CM = International Classification of Diseases, Ninth Revision, Clinical Modification; ITBP = intrathecal baclofen pump; LOS = length of stay; PHIS = Pediatric Health Information System; RR = relative risk; SCIP = Surgical Care Improvement Project; SSI = surgical-site infection; USD = US dollars.

SUBMITTED August 1, 2015. ACCEPTED September 11, 2015.

INCLUDE WHEN CITING DOI: 10.3171/2015.9.FOCUS15385. 
$\mathrm{T}$ He intrathecal baclofen pump (ITBP) was first introduced for treatment of spasticity of spinal origin. ${ }^{13}$ The surgery involves the implantation of a permanent intrathecal catheter, reservoir, and pump mechanism to allow instillation of medication. Infections are not uncommon with these surgeries, which involve the presence of implants in a patient population with complex medical conditions. Infection rates were reported in some studies to be $4.5 \%-9 \%$ or even higher in children $(9 \%-12 \%) .3 .5 \mathrm{~A}$ collaborative study among European countries for consensus on the appropriate use of ITBP in the pediatric population was published in $2010 .{ }^{3}$ Few studies have investigated postoperative complication and infection rates of ITBP in the pediatric population, and findings are limited., ${ }^{4,10,15}$ Increased mechanical complications between 1997 and 2006 were reported in 1 study. ${ }^{15}$

Surgical-site infections (SSI) accounted for $31 \%$ of all hospital-acquired infection among hospitalized patients, according to a report by Magill et al., ${ }^{8}$ and the overall rate of SSI reported by the National Healthcare Safety Network was $1.9 \% .^{11}$ The consequences of inappropriate use of antibiotics have also been reported to increase the risk of antimicrobial resistance and hospital-acquired infections and complications. ${ }^{2,12,16}$ For a patient population with chronic healthcare needs, such as children needing ITBP therapy, risk of surgery-related infection as well as overall healthcare burden is high.

The clinical practice guideline for antimicrobial prophylaxis in surgery was updated by the American Society of Health-System Pharmacists (ASHP) in 2013. ${ }^{2}$ These guidelines were developed jointly by the ASHP, the Infectious Diseases Society of America, the Surgical Infection Society, and the Society of Healthcare Epidemiology of America. The ASHP guidelines are intended to provide practitioners with an evidence-based standardized approach for use of antimicrobial agents for the prevention of SSI. Emerging issues such as antimicrobial resistance were also considered. A single dose of cefazolin is recommended for patients undergoing intrathecal pump placement, revision, or replacement. Clindamycin or vancomycin is reserved as an alternative agent for patients with a documented $\beta$-lactam allergy. The recommended dosages for pediatric patients treated with cefazolin, clindamycin, and vancomycin are $30 \mathrm{mg} / \mathrm{kg}$ every 4 hours, $10 \mathrm{mg} / \mathrm{kg}$ every 6 hours, and $15 \mathrm{mg} / \mathrm{kg}$, with no recommended redosing interval, respectively. The recommended timing for perioperative dosing is within 60 minutes before surgical incision. A postoperative course of antimicrobials continuing for less than 24 hours is recommended.

We conducted a study with the following 3 aims: 1) to understand the utilization of perioperative antibiotics for ITBP surgery in US freestanding pediatric hospitals, 2) to investigate the documented compliance with clinical practice guidelines of perioperative antibiotic administration for ITBP surgery and the factors associated with compliance, 3) to explore the association between antibiotic prophylaxis (AP) compliance and outcomes measures of cost of ITBP surgical hospitalization and 6-month surgical complication/infection.

\section{Methods \\ Data Source}

The Children's Hospital Association's Pediatric Health Information System (PHIS) data were used in this study. PHIS contains inpatient, emergency department, ambulatory surgery, and observation encounter level data from 43 freestanding children's hospitals. PHIS data included patient demographics, detailed charges, and treatment information. All encounter-level data are de-identified. This study received exempt status from the Baylor College of Medicine Institutional Review Board.

\section{Study Cohort}

Inclusion criteria included patients who had an ITBP procedure within 3 days of admission. As in prior study designs, ${ }^{9,18}$ we avoided complicated cases associated with long hospitalizations prior to ITBP surgery. Patients were discharged between July 1, 2004, and March 31, 2014, and had a minimum of 180 days of prior screening in the database along with a minimum 180-day follow-up period. Age range was 0-17 years. International Classifcation of Diseases, Ninth Revision, Clinical Modification (ICD-9-CM) diagnoses and procedures codes were used to identify the ITBP study cohort, which was defined by ICD-9-CM procedure code 86.06 (insertion of totally implantable infusion pump) along with 1 of the following diagnoses: hereditary spastic paraplegia, spastic hemiplegia, unspecified hemiplegia, congenital diplegia, congenital hemiplegia, congenital quadriplegia, infantile hemiplegia, cerebral palsy, quadriplegia unspecified, other quadriplegia, paraplegia, anoxic brain damage, muscle spasm, and abnormal involuntary movements (ICD-9-CM diagnosis codes: $334.1,342.10-342.92,343.0,343.1,343.2$, 343.4, $343.8,343.9,344.00,344.09,344.1,728.85,781.0)$. We excluded patients who had missing inpatient financial data, prior documented infections or complications, and/or recorded antibiotic administration within 30 days of admission for surgery. Table 1 provides patient characteristics and factors associated with overall bundled compliance.

There was neither exact perioperative drug administration timing nor reliable dosage information in the PHIS database. Patients were given credit for AP if antibiotic administration was documented at Day 0 (day of surgery), or both Day 0 and Day 1 (day of surgery and postoperative Day 1). Overall bundled AP compliance was defined according to AHSP guidelines in the study; in this PHIS dataset, we captured administration of antibiotics on day of surgery and looked for discontinuation of the antibiotic the day after surgery. Continuation beyond a 24-hour period was not considered compliant with AHSP guidelines. Additionally, we examined each component of compliance separately: 1) preoperative antibiotics were not given (missed preoperative antibiotic), and 2) preoperative antibiotics were given, but antibiotic administration continued past 24 hours postoperatively (prolonged antibiotic administration). The costs per ITBP procedure were calculated with PHIS-defined adjusted total costs, based upon the ratio of cost to charges submitted by the hospitals with their 
TABLE 1. Patient characteristics associated with overall bundled compliance

\begin{tabular}{|c|c|c|c|c|c|c|c|}
\hline \multirow[b]{2}{*}{ Characteristic } & \multirow[b]{2}{*}{ No. of Cases } & \multirow[b]{2}{*}{$\%$} & \multirow[b]{2}{*}{ Compliance } & \multirow[b]{2}{*}{$x^{2} p$ Value } & \multicolumn{3}{|c|}{ Overall Bundled Compliance } \\
\hline & & & & & OR & $95 \% \mathrm{Cl}$ & Logistic Regression $p$ Value* \\
\hline Total & 1534 & & 64.2 & & & & \\
\hline Age (yrs) & & & & 0.323 & & & \\
\hline $0-4$ & 154 & 10.0 & 69.5 & & & & \\
\hline $5-9$ & 553 & 36.1 & 62.9 & & & & \\
\hline $10-17$ & 827 & 53.9 & 64.1 & & & & \\
\hline Sex & & & & 0.163 & & & $0.111 \dagger$ \\
\hline $\mathrm{F}$ & 610 & 39.8 & 62.0 & & 1.00 & & \\
\hline M & 924 & 60.2 & 65.7 & & 1.19 & $0.96-1.48$ & 0.111 \\
\hline Race & & & & 0.013 & & & $0.013 \dagger$ \\
\hline White & 958 & 62.5 & 66.9 & & 1.00 & & \\
\hline Black & 244 & 15.9 & 58.6 & & 0.74 & $0.55-1.00$ & 0.052 \\
\hline Hispanic & 227 & 14.8 & 57.7 & & 0.63 & $0.47-0.86$ & 0.004 \\
\hline Others $\ddagger$ & 105 & 6.8 & 66.7 & & 1.02 & $0.65-1.59$ & 0.946 \\
\hline Insurance & & & & 0.530 & & & \\
\hline Private & 476 & 31.0 & 63.7 & & & & \\
\hline Public & 887 & 57.8 & 65.2 & & & & \\
\hline Others & 171 & 11.2 & 60.8 & & & & \\
\hline Region & & & & 0.001 & & & $<0.001 \uparrow$ \\
\hline Northeast & 221 & 14.4 & 55.2 & & 1.00 & & \\
\hline Midwest & 365 & 23.8 & 66.0 & & 1.62 & $1.14-2.30$ & 0.007 \\
\hline South & 532 & 34.7 & 62.0 & & 1.47 & $1.06-2.03$ & 0.021 \\
\hline West & 416 & 27.1 & 70.2 & & 2.04 & $1.44-2.88$ & $<0.001$ \\
\hline Diagnosis & & & & 0.439 & & & \\
\hline Cerebral palsy & 1193 & 77.8 & 63.7 & & & & \\
\hline Others & 341 & 22.2 & 66.0 & & & & \\
\hline Admission yr & & & & $<0.001$ & & & $0.001 \dagger$ \\
\hline Pre-2013 & 1409 & 91.9 & 62.5 & & 1.00 & & \\
\hline 2013-2014 & 125 & 8.15 & 78.4 & & 2.18 & $1.38-3.44$ & 0.001 \\
\hline
\end{tabular}

\footnotetext{
* Hosmer-Lemeshow goodness of fit and link test showed the model fit well.

$\dagger$ Joint significance of the covariate; higher odds mean higher chances of compliance.

$\ddagger$ Less than 10 patients with "unknown" race.
}

respective Medicare cost reports. Charges were adjusted by the Centers of Medicare and Medicaid wage/price index for the hospital's location. All costs were converted to 2009 US dollars (USD). Six-month postoperative complication and infection rates were recorded. All complication and infection events were identified through ICD-9-CM diagnosis codes: 996.2X, 996.6X, 996.7X, 997.0X, 997.3X, 998.0X, 998.1X-998.4X, 998.7X-998.9X, and 998.5X.15,18

\section{Statistical Analysis}

Chi-square tests and multivariate logistic regression models were used to determine factors associated with overall bundled compliance with prophylactic antibiotic administration and proper antibiotic duration in ITBP surgery. A multivariate regression model was used to determine the association between ITBP hospitalization costs and the compliance to AP. We also performed multinomial logistic regression for the noncompliant subgroups: missed preoperative antibiotic and prolonged antibiotic administration. Any covariate in univariate analysis with $\mathrm{p}$ value $<0.2$ was included in the multivariate logistic regression model or in multivariate regression model. Analyses were conducted with SAS 9.4 (SAS Institute) and STATA 13.0 software (StataCorp).

\section{Results}

A total of 1,534 patients met inclusion criteria (Fig. 1); 91.5\% received AP. Among those, $34.6 \%$ received 2 antibiotics. The most frequently used antibiotics on the day of surgery were cefazolin $(\mathrm{n}=873,61.96 \%)$ and vancomycin $(\mathrm{n}=351,24.91 \%)$, in keeping with guideline recommendations. Around two-thirds of ITBP surgeries (64.2\%) were compliant with practice guidelines in overall bundled compliance, including appropriate timing and $<24$-hour duration of administration of AP. Documented AP administration compliance rates were $70.2 \%, 62.0 \%, 66.0 \%$, and $55.2 \%$ in West, South, Midwest, and Northeast regions of the US, respectively. Non-Hispanic white patients had the highest documented overall bundled AP compliance 


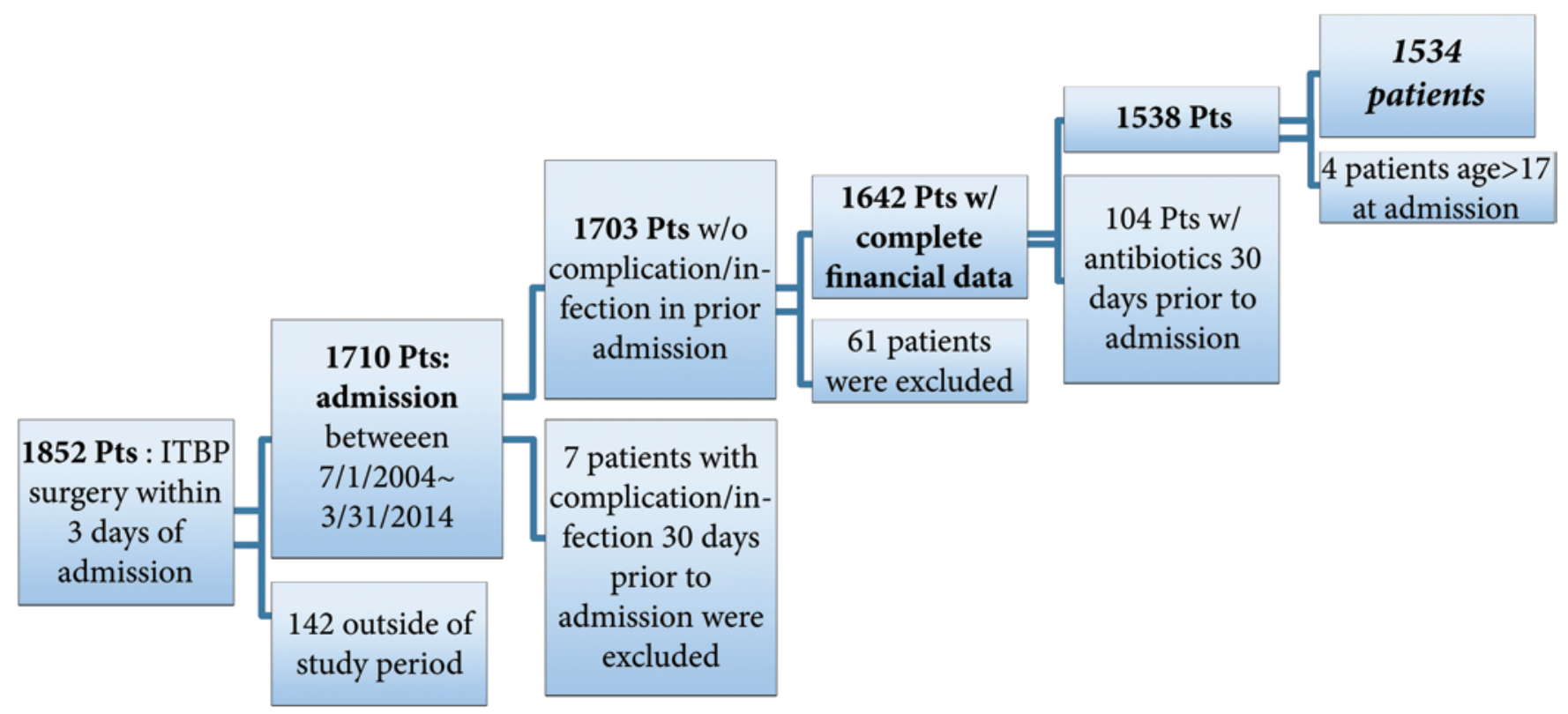

FIG. 1. Consortium chart showing derivation of the study cohort. Pts $=$ patients.

rates (66.9\%), compared with non-Hispanic black (58.6\%) and Hispanic (57.7\%) patients. In the multivariate logistic regression model, we included sex $(p=0.163)$, race $(p=$ $0.013)$, and region $(\mathrm{p}=0.001)$. While controlling for other covariates, compared with surgeries in the Northeast, those in the West (OR 2.0, 95\% CI 1.4-2.8, p < 0.001), Midwest (OR 1.6, 95\% CI 1.1-2.3, $\mathrm{p}=0.008)$, and South (OR $1.4,95 \%$ CI 1.0-2.0, $\mathrm{p}=0.029$ ) were more likely to have documented overall bundled AP compliance. Black (OR 0.73, 95\% CI 0.55-0.99, $\mathrm{p}=0.039)$ and Hispanic (OR $0.64,95 \%$ CI $0.47-0.88, \mathrm{p}=0.005$ ) patients were less likely to have overall bundled AP compliance for their procedures than white patients. No significant variations in compliance rates were shown in subgroups of age, sex, type of insurance, and diagnosis.

Length of stay (LOS), adjusted costs of ITBP hospitalization, and 6-month postsurgical infection and complication rates differed significantly between the groups with documented AP compliance and AP noncompliance (Fig. 2). The compliant group with the overall AP bundle had slightly lower postsurgical infection and complication rates within 6 months following surgery than the noncompliant group $(20.00 \%$ and $24.23 \%$, respectively, $p$ value $=$ $0.054)$. The mean LOS in the compliant group was 3.45 days (95\% CI 3.50-3.88 days); the adjusted costs of ITBP procedures in 2009 USD were an average of $\$ 22,828$ USD (95\% CI \$20,595-\$25,060 USD).

The overall bundled AP compliant group tended to have shorter LOS and lower costs than the noncompliant group (mean $\operatorname{LOS}=4.2$ days, 95\% CI 3.79-4.61 days; mean adjusted costs in 2009 USD $=\$ 34,281$ USD, 95\% CI: $\$ 27,689-\$ 40,873$ USD) (Fig. 2 upper). In addition, compared with patients who had ITBP placement in the Northeast, patients who underwent ITBP surgery in the South and West (both $\mathrm{p}<0.001$ ) were more likely to have lower hospitalization costs.

Subgroup analysis examined outcomes with the 2 sub- types of noncompliance: missed preoperative antibiotic and prolonged antibiotic administration (preoperative antibiotic given but continued past 24 hours postoperatively) (Table 2). Compliance rates in both subgroups were affected by similar factors, in time period, race, and region. In the multivariate cost regression model (Table 3), we included overall bundled compliance, age, and region and found that patients with overall bundled compliance to the ASHP guidelines were more likely to incur less cost ( $\mathrm{p}<$ 0.001).

There were no differences in subgroup compliance by sex. There was significant improvement in each subgroup process measure over time, pre-2013 compared with 2013-2014. Hispanic race was a significant risk factor for missed preoperative antibiotic (relative risk [RR] $1.68, \mathrm{p}=0.016$ ) while both black and Hispanic race were risk factors for prolonged antibiotic duration (RR 1.43, $\mathrm{p}$ $=0.045$ and RR 1.52, p = 0.024). Regional variation was found for both subgroups, which showed significantly lower noncompliance with preoperative antibiotic administration in the Midwest (RR 0.59, p = 0.02), South ( RR $0.43, \mathrm{p}<0.001$ ), and West (RR 0.34, p < 0.001) regions compared with the Northeast. For proper $<24$-hour duration of postoperative prophylactic antibiotic use, the Midwest (RR 0.64, $\mathrm{p}=0.046)$ and West (RR 0.065, $\mathrm{p}=0.043$ ) regions documented less noncompliance when compared with the Northeast. Subgroup compliance was also associated with health care utilization and outcomes (Fig. 2 lower). There was a difference ( $\mathrm{p}<0.001)$ in LOS between groups with overall bundled compliance, missed preoperative antibiotic, and prolonged antibiotic administration (3.19, 5.00, 4.08 days, respectively). Significant differences $(p<0.001)$ also existed between the 3 above compliance categories for adjusted costs of surgical hospitalization $(\$ 22,828,95 \%$ CI $\$ 20,595-\$ 25,060 ; \$ 29,103,95 \%$ CI $\$ 24,243-\$ 33,965$; and $\$ 37,663,95 \%$ CI $\$ 27,228-\$ 48,099$, respectively). This pattern was also confirmed in the mul- 
Length of Stay, Adjusted Costs, and 6-Month Infection/Complication Rates of ITBP by AP Compliance

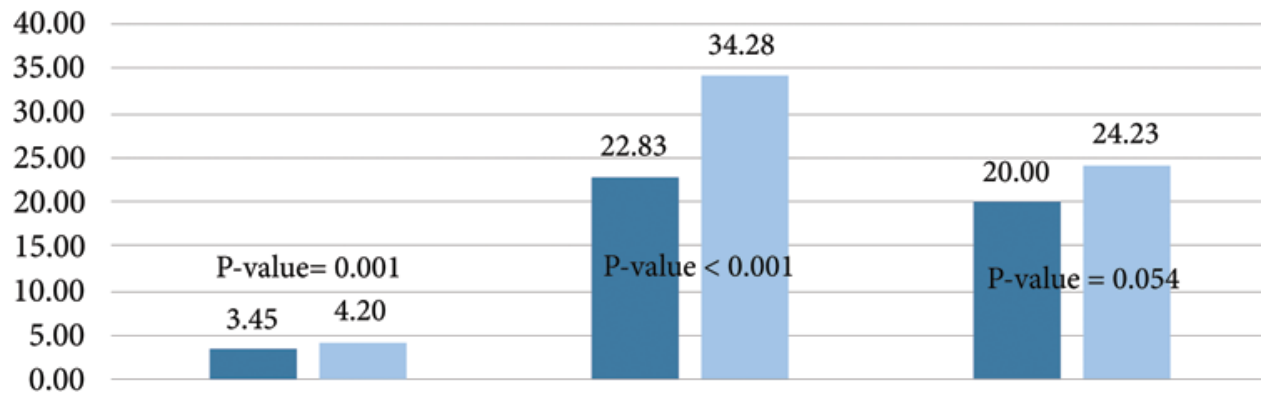

Length of Stay (Days) Adjusted Costs (Thousands) Infection/Complication rates

- Overall bundled compliance $\quad$ Noncompliant with bundle

$(\%)$

Length of Stay, Adjusted Costs, and 6-Month Infection/Complication Rates of ITBP by Subgroup Compliance

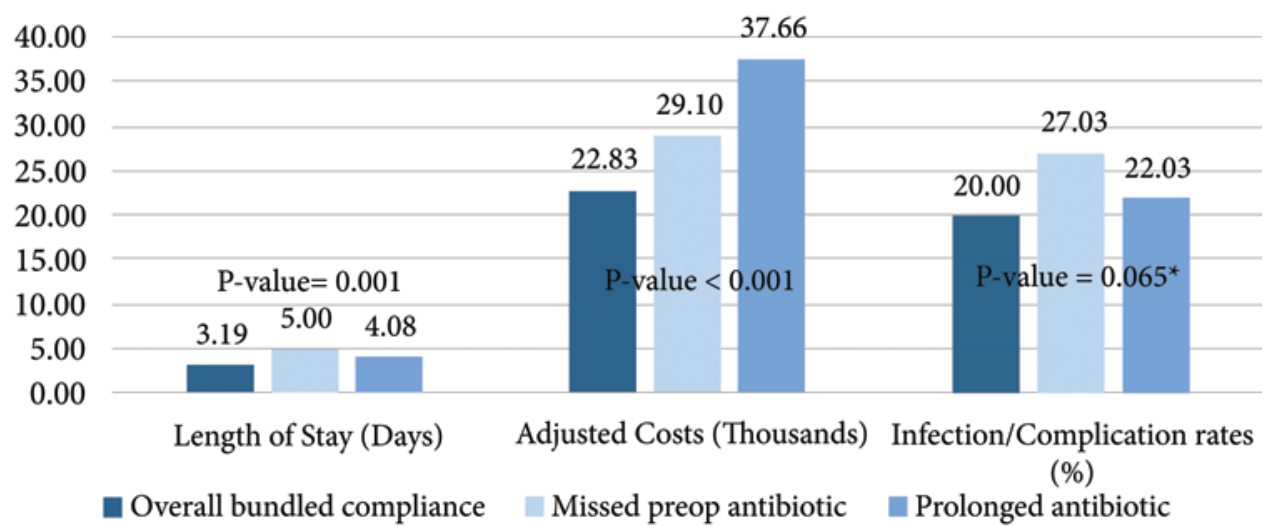

FIG. 2. LOS, adjusted costs, and 6-month infection/complication rates of ITBP by AP compliance (upper) and by subgroup compliance (lower). *Single logistic regression showed that the missed preoperative antibiotic group had a significantly higher rate of 6 -month surgical complication/infection compared with the overall bundled compliance group $(27.03 \%$ vs $20.00 \%, p=0.021,0 R$ $1.48,95 \% \mathrm{Cl} 1.06-2.07)$. The other subgroup, prolonged antibiotic administration, did not differ significantly $(22 \%, p=0.368,0 R$ $1.15,95 \% \mathrm{Cl} 0.85-1.56)$.

tivariate model (Table 3), where the prolonged antibiotic administration subgroup was associated with the highest cost. Differences in infection/complication rates did not quite reach statistical significance $(\mathrm{p}=0.065)$ when 3 groups were compared all together, with rates of $20.00 \%$, $27.03 \%$, and $22.03 \%$ for overall bundle compliant, missed preoperative antibiotic, and prolonged antibiotic administration groups, respectively. However, most strikingly, the missed preoperative antibiotic group had statistically significantly higher 6-month surgical infection/complication rates $(27.03 \%)$ when compared with the overall bundlecompliant group (20.00\%). The prolonged antibiotic use group had a $22.03 \%$ 6-month surgical infection/complication rate and did not significantly differ from the overall bundled group.

\section{Discussion}

We examined antibiotic prophylaxis compliance with clinical practice guidelines in antimicrobial prophylaxis in pediatric intrathecal baclofen pump surgeries. Two- thirds of the ITBP surgeries (64.2\%) were documented as compliant with overall bundled perioperative AP administration guidelines including timing and duration of therapy. Compliance rates in the study period prior to 2013 were $62.95 \%$ and jumped to $78.4 \%$ in 2013 and 2014. This increase in detected overall bundled AP compliance rates as well as preoperative antibiotic and $<24$-hour AP duration rates may stem from increased antibiotic administration or documentation, adherence to 2013 guidelines, or may include systems enhancements, policy changes, or performance incentives for administration or documentation of perioperative prophylactic antibiotic practices. The development and influence of antibiotic stewardship programs at participating hospitals may also play a role. Further study is needed to understand the underlying influences responsible for these changes.

Previous studies have shown variation in practice patterns in pediatric hospitals. ${ }^{9}$ We observe geographic and practice variation in this study as well. Highest rates of documented AP compliance were in the West region. Intravenous perioperative antibiotics are typically less an 
TABLE 2. Multinomial logistic regression model of compliance (analysis of subgroups: missed preoperative antibiotic and prolonged antibiotic use)

\begin{tabular}{|c|c|c|c|}
\hline Compliance Subgroup \& Covariate & RR Ratio & $95 \% \mathrm{Cl}$ & $\mathrm{p}$ Value \\
\hline \multicolumn{4}{|l|}{ Missed preop antibiotic } \\
\hline Sex & & & $0.284^{*}$ \\
\hline $\mathrm{F}$ & 1.00 & & \\
\hline $\mathrm{M}$ & 0.85 & $0.63-1.15$ & 0.284 \\
\hline Race & & & $0.066^{*}$ \\
\hline White & 1.00 & & \\
\hline Black & 1.22 & $0.81-1.85$ & 0.338 \\
\hline Hispanic & 1.68 & $1.10-2.57$ & 0.016 \\
\hline Others & 0.80 & $0.41-1.55$ & 0.504 \\
\hline Region & & & $<0.001^{*}$ \\
\hline Northeast & 1.00 & & \\
\hline Midwest & 0.59 & $0.38-0.92$ & 0.020 \\
\hline South & 0.43 & $0.28-0.67$ & $<0.001$ \\
\hline West & 0.34 & $0.21-0.54$ & $<0.001$ \\
\hline Admission yr & & & $0.019^{*}$ \\
\hline Pre-2013 & 1.00 & & \\
\hline 2013-2014 & 0.45 & $0.23-0.88$ & 0.019 \\
\hline \multicolumn{4}{|l|}{ Prolonged antibiotic use } \\
\hline Sex & & & $0.165 \dagger$ \\
\hline $\mathrm{F}$ & 1.00 & & \\
\hline $\mathrm{M}$ & 0.83 & $0.64-1.08$ & 0.165 \\
\hline Race & & & $0.056 \dagger$ \\
\hline White & 1.00 & & \\
\hline Black & 1.43 & $1.01-2.02$ & 0.045 \\
\hline Hispanic & 1.52 & $1.06-2.18$ & 0.024 \\
\hline Others & 1.12 & $0.67-1.87$ & 0.670 \\
\hline Region & & & $0.028 \dagger$ \\
\hline Northeast & 1.00 & & \\
\hline Midwest & 0.64 & $0.41-0.99$ & 0.046 \\
\hline South & 0.94 & $0.63-1.39$ & 0.750 \\
\hline West & 0.65 & $0.42-0.99$ & 0.043 \\
\hline Admission yr & & & $0.007 \dagger$ \\
\hline \multicolumn{4}{|l|}{ Pre-2013 } \\
\hline 2013-2014 & 0.46 & $0.27-0.81$ & 0.007 \\
\hline
\end{tabular}

* Joint significance of the covariate in missed preoperative antibiotic equation. $\dagger$ Joint significance of the covariate in prolonged antibiotic equation.

issue of patient compliance and may be related more to provider practice, documentation/capture, or institutional processes. No significant variations in overall or subgroup compliance rates were shown in subgroups of age, sex, type of insurance, and diagnosis, though there were significant differences in AP compliance by race. Findings of racial disparity in pediatric surgical care are not unique, ${ }^{1,67}$ reasons for this disparity in AP compliance are unclear and warrant further study. As substratification with specific patient comorbidities was not conducted in this study, it may be that there were specific clinical concerns or indications requiring prolonged antibiotic use beyond the recommended $<24$-hour duration in guidelines. Patients who underwent a prolonged duration of antibiotic administration did not have a significantly higher or lower surgical complication/infection rate compared with overall bundlecompliant patients, though costs were significantly higher.

Process measures such as perioperative antibiotics and antibiotic choice have been examined across surgical specialties and have not been uniformly shown to reflect on outcome. For example, implementation of Surgical Care Improvement Project (SCIP) measures in adult surgeries led to improved documented compliance rates with SCIP measures but did not necessarily yield improved outcomes in reduction of infections. ${ }^{14,17}$ In this study, SCIP measures of preoperative antibiotic administration indeed correlated with improved outcomes in this pediatric population undergoing intrathecal baclofen pump surgeries. There was a significant difference between patients with documented overall bundled compliance with antibiotic prophylaxis and those without documented compliance. Each component of antibiotic prophylaxis practice, including preoperative antibiotic administration and $<24$-hour duration of postoperative antibiotic use, shows measurable impact on healthcare utilization and outcome. The subgroup of patients using prolonged antibiotics had higher hospitalization costs $(\$ 37,663,95 \%$ CI $\$ 27,228-\$ 48,099)$ than those who missed preoperative antibiotics $(\$ 29,103,95 \%$ CI $\$ 24,243-\$ 33,965)$, who in turn had higher costs than those with overall bundled AP compliance $(\$ 22,830,95 \%$ CI $\$ 20,595-\$ 25,060)$. This practice variation shows a wide range in costs and presents opportunities for improvement. Prolonged antibiotic use did not appear to confer any advantage in outcomes; 6-month surgical complication/infection rates associated with prolonged antibiotics $(22.00 \%)$ compared with overall bundled compliance $(20.00 \%)$ did not differ significantly $(p=0.368)$. In addition to increased costs, prolonged antibiotic use may have greater implications for antibiotic stewardship and public health, though the consideration of these factors are beyond the scope of this study. $2,12,16$ Perhaps the single most compelling and practical finding for clinicians pertains to administration of preoperative antibiotics: the subgroup of patients who missed preoperative antibiotic administration had a significantly higher 6-month surgical infection/complication (rate $27.03 \%$, OR 1.48 , p value $=0.021$ ) compared with patients who received the overall AP bundle (rate 20.03\%). While our results have multiple avenues for examining quality, this striking finding involving the administration of preoperative antibiotic is directly linked to surgical outcomes and impacts clinical practice. This result reinforces preoperative antibiotic administration as the standard of care.

Overall bundled AP compliance was associated with a shorter LOS and lower adjusted costs of the ITBP hospitalization. There was also a trend toward significance with lower 6-month postsurgical infection and complication rates. Higher documented AP compliance thus appears in this study to be associated with the delivery of a higher value of care, with better quality of care, and with lower costs. The reasons for these study findings need to be explored in future study directions: the effect of practice variation, institutional policies, surgical volume, surgeon practice, multidisciplinary care programs, surgical team infrastructure, and medical homes may be considered. 
TABLE 3. Factors associated with adjusted hospitalization costs for intrathecal baclofen pump procedure

\begin{tabular}{|c|c|c|c|c|c|c|}
\hline \multirow[b]{2}{*}{ Factor } & \multicolumn{3}{|c|}{ Compliance With Overall Bundled AP Use } & \multicolumn{3}{|c|}{ Compliance With AP Use Subgroups } \\
\hline & Coefficient & $95 \% \mathrm{Cl}$ & p Value & Coefficient & $95 \% \mathrm{Cl}$ & p Value \\
\hline Compliance, reference & 0.000 & & & 0.000 & & \\
\hline Noncompliance & 0.138 & $0.071-0.204$ & $<0.001$ & NA & NA & \\
\hline Missed preop antibiotic & NA & NA & & 0.100 & $0.002-0.197$ & 0.045 \\
\hline Prolonged antibiotic use & NA & NA & & 0.195 & $0.115-0.275$ & $<0.001$ \\
\hline \multicolumn{7}{|l|}{ Age, yrs } \\
\hline $0-4$, reference & 0.000 & & & 0.000 & & \\
\hline $5-9$ & 0.093 & $0.007-0.178$ & 0.034 & 0.094 & $0.011-0.178$ & 0.027 \\
\hline $10-17$ & 0.116 & $0.034-0.198$ & 0.005 & 0.111 & $0.032-0.189$ & 0.006 \\
\hline \multicolumn{7}{|l|}{ Region } \\
\hline Northeast & 0.000 & & & 0.000 & & \\
\hline Midwest & 0.085 & $-0.014-0.185$ & 0.094 & 0.095 & $-0.001-0.192$ & 0.054 \\
\hline South & -0.230 & -0.304 to -0.157 & $<0.001$ & -0.235 & -0.307 to -0.163 & $<0.001$ \\
\hline West & -0.274 & -0.351 to -0.196 & $<0.001$ & -0.296 & -0.372 to -0.219 & $<0.001$ \\
\hline
\end{tabular}

$\mathrm{NA}=$ not applicable.

\section{Limitations}

Our study was limited by factors inherent to the retrospective analysis of a large database. Data are subject to coding or reporting bias. Rates of antibiotic prophylaxis appear low in this study: we are unable to distinguish between lack of antibiotic administration or lack of documentation when antibiotics were actually given. We are reliant on documentation in the database as well as diagnosis and procedural ICD-9 coding. Surgical technique is not known. We tested the identification of ITBP procedures as well as the diagnosis of pump infection and device complication with an internal validation at our institution; this showed high sensitivity, specificity, and positive predictive values of $>90 \%$. It is difficult to distinguish between codes for placement and revision, catheter manipulation or pump replacement: all ITBP surgeries were thus considered together. Further multicenter validation is necessary in the future. Antibiotics intended for prophylaxis were selected as those given on the day of surgery, with strict exclusion criteria, though exact timing of administration on the day of surgery in relation to time of surgical incision is not known. We are not able to identify other variables-such as operating room sterility practices, intrawound antibiotic use, distant history of prior infections (as our minimum prior screening time was 6 months), or results of methicillin-resistant Staphylococcus aureus screening. Despite these limitations, our study provides a unique assessment of prophylactic antibiotic use in intrathecal baclofen pump surgeries in children. A notable strength of this study is that PHIS data provides a large sample size from across a multicenter representation of 43 pediatric hospitals of the Children's Hospital Association. Unlike national hospital discharge datasets such as the Kids' Inpatient Database, PHIS captures longitudinal data. Preoperative and postoperative longitudinal inclusion and exclusion criteria can be applied, and follow-up for downstream events and utilization within each hospital can be measured. Important antibiotic stewardship considerations and greater epide- miological impact are not accounted for in this study and warrant further exploration.

\section{Conclusions}

Geographic, race, and practice variation in administration of antibiotic prophylaxis in intrathecal baclofen pump procedures were found in this study. Antibiotic prophylaxis in ITBP procedures was associated with higher value of care, showing positive clinical and financial outcomes. Of most impact to clinical outcome, missed preoperative antibiotics was significantly associated with higher risk 6-month surgical complication/infection. Prolonged antibiotic use was associated with significantly higher hospital costs compared with those with overall bundled antibiotic compliance. Future research is warranted to examine factors associated with these practice variations, and to examine how antibiotic prophylaxis practice is associated with outcomes and quality, aiming for improving overall delivery of care to pediatric patients undergoing ITBP procedures.

\section{References}

1. Attenello FJ, Ng A, Wen T, Cen SY, Sanossian N, Amar AP, et al: Racial and socioeconomic disparities in outcomes following pediatric cerebrospinal fluid shunt procedures. J Neurosurg Pediatr 15:560-566, 2015

2. Bratzler DW, Dellinger EP, Olsen KM, Perl TM, Auwaerter PG, Bolon MK, et al: Clinical practice guidelines for antimicrobial prophylaxis in surgery. Am J Health Syst Pharm 70:195-283, 2013

3. Dan B, Motta F, Vles JS, Vloeberghs M, Becher JG, Eunson $\mathrm{P}$, et al: Consensus on the appropriate use of intrathecal baclofen (ITB) therapy in paediatric spasticity. Eur J Paediatr Neurol 14:19-28, 2010

4. Dickey MP, Rice M, Kinnett DG, Lambert R, Donauer S, Gerber MA, et al: Infectious complications of intrathecal baclofen pump devices in a pediatric population. Pediatr Infect Dis J 32:715-722, 2013 
5. Fjelstad AB, Hommelstad J, Sorteberg A: Infections related to intrathecal baclofen therapy in children and adults: frequency and risk factors. J Neurosurg Pediatr 4:487-493, 2009

6. Lam SK, Pan IW, Harris DA, Sayama CM, Luerssen TG, Jea A: Patient-, procedure-, and hospital-related risk factors of allogeneic and autologous blood transfusion in pediatric spinal fusion surgery in the United States. Spine (Phila Pa 1976) 40:560-569, 2015

7. Lin Y, Pan IW, Harris DA, Luerssen TG, Lam S: The impact of insurance, race, and ethnicity on age at surgical intervention among children with nonsyndromic craniosynostosis. J Pediatr 166:1289-1296, 2015

8. Magill SS, Hellinger W, Cohen J, Kay R, Bailey C, Boland B, et al: Prevalence of healthcare-associated infections in acute care hospitals in Jacksonville, Florida. Infect Control Hosp Epidemiol 33:283-291, 2012

9. McLeod LM, Keren R, Gerber J, French B, Song L, Sampson NR, et al: Perioperative antibiotic use for spinal surgery procedures in US children's hospitals. Spine (Phila Pa 1976) 38:609-616, 2013

10. Motta F, Buonaguro V, Stignani C: The use of intrathecal baclofen pump implants in children and adolescents: safety and complications in 200 consecutive cases. J Neurosurg 107 (1 Suppl):32-35, 2007

11. Mu Y, Edwards JR, Horan TC, Berrios-Torres SI, Fridkin SK: Improving risk-adjusted measures of surgical site infection for the national healthcare safety network. Infect Control Hosp Epidemiol 32:970-986, 2011

12. Ohl CA, Luther VP: Antimicrobial stewardship for inpatient facilities. J Hosp Med 6 (Suppl 1):S4-S15, 2011

13. Penn RD, Kroin JS: Continuous intrathecal baclofen for severe spasticity. Lancet 2:125-127, 1985

14. Stulberg JJ, Delaney CP, Neuhauser DV, Aron DC, Fu P, Koroukian SM: Adherence to surgical care improvement project measures and the association with postoperative infections. JAMA 303:2479-2485, 2010

15. Varhabhatla NC, Zuo Z: Rising complication rates after intra- thecal catheter and pump placement in the pediatric population: analysis of national data between 1997 and 2006. Pain Physician 15:65-74, 2012

16. Veličković-Radovanović R, Stefanović N, Damnjanović I, Kocić B, Antić S, Dinić M, et al: Monitoring of antibiotic consumption and development of resistance by enterobacteria in a tertiary care hospital. J Clin Pharm Ther 40:426-430, 2015

17. Wang Z, Chen F, Ward M, Bhattacharyya T: Compliance with Surgical Care Improvement Project measures and hospital-associated infections following hip arthroplasty. J Bone Joint Surg Am 94:1359-1366, 2012

18. Warren DK, Nickel KB, Wallace AE, Mines D, Fraser VJ, Olsen MA: Can additional information be obtained from claims data to support surgical site infection diagnosis codes? Infect Control Hosp Epidemiol 35 (Suppl 3):S124-S132, 2014

\section{Disclosures}

The authors report no conflict of interest concerning the materials or methods used in this study or the findings specified in this paper.

\section{Author Contributions}

Conception and design: Pan. Acquisition of data: Pan. Analysis and interpretation of data: Pan, Kuo. Drafting the article: Lam, Pan. Critically revising the article: all authors. Reviewed submitted version of manuscript: Lam, Pan. Approved the final version of the manuscript on behalf of all authors: Lam. Statistical analysis: Pan. Administrative/technical/material support: Lam, Pan, Luerssen. Study supervision: Lam, Pan.

\section{Correspondence}

Sandi K. Lam, Department of Neurosurgery, Texas Children's Hospital, 6701 Fannin St., CCC 1230.01, Houston, TX 77030. email: sklam@texaschildrens.org. 